\title{
Fractal Weyl laws for quantum decay in dynamical systems with a mixed phase space
}

\author{
Marten Kopp and Henning Schomerus \\ Department of Physics, Lancaster University, Lancaster LA1 4YB, United Kingdom \\ (Received 10 July 2009; revised manuscript received 7 January 2010; published 19 February 2010)
}

\begin{abstract}
Weyl's law approximates the number of states in a quantum system by partitioning the energetically accessible phase-space volume into Planck cells. Here, we show that resonances in open quantum systems can follow a modified fractal Weyl law, even when their classical dynamics is not globally chaotic but also contains domains of regular motion. Using an appropriate phase-space representation for open quantum systems, we connect this behavior to emerging quantum-to-classical correspondence.
\end{abstract}

DOI: 10.1103/PhysRevE.81.026208

\section{INTRODUCTION}

Phase-space rules provide powerful universal relations for classical and quantum systems. A time-honored example is Sabine's law, originally formulated in the context of room acoustics, which can be cast into the relation $\tau_{\text {dwell }}=4 \mathrm{~V} / \mathrm{vA}$ for the mean dwell time of a classical particle escaping from a container, expressed in terms of the volume $V$ of the container, the area $A$ of the opening, and the particle's velocity $v$ [1]. In quantum mechanics, Weyl's law approximates the number $\mathcal{N}(E)$ of states with energy $E_{n}<E$ by the number of Planck cells $h^{d}$ which fit into the accessible phase-space volume of the corresponding classical system (here $h$ is the Planck's constant and $d$ is the number of dimensions) [2]. For a quantum particle in a container (e.g., an electron in a quantum dot, with $h$ fixed $), \mathcal{N}(E) \propto E^{d / 2}$ therefore follows a power law with a strictly determined exponent.

In reality, quantum systems are open, and the eigenstates acquire a finite lifetime - the resulting resonance states constitute a fundamental concept across many fields of physics. However, there are reasons to believe that the synthesis of both mentioned phase-space rules in open quantum systems can modify Weyl's law. Evidence in this direction is provided by systems with globally chaotic classical dynamics, which exhibit a fractal Weyl law $\mathcal{N}(E, \tau) \propto c_{\tau} E^{d_{H} / 2}$ for the number of resonances with $E_{n}<E$ and lifetime $\tau_{n}>\tau$ (where the cutoff value $\tau$ only enters the shape function $c_{\tau}$ ) [3-6]. Remarkably, the number $d_{H}$ in the exponent is not an integer; instead, it is given by the dimension of the strange repeller, which for a chaotic system is a fractal [7]. While this observation already led to a paradigm shift of the understanding of resonances in open quantum-chaotic systems, its direct practical consequences are necessarily limited: generic dynamical systems are not globally chaotic, which has profound consequences for their quantum dynamics [8].

Here, we show that fractal Weyl laws indeed apply much more generally also to dynamical systems for which regular and chaotic motions coexist in a mixed phase space [7]. The key which reveals the fractal Weyl law is to restrict the resonance counting to a window of intermediate lifetimes $\left(\tau<\tau_{n}<\tau^{\prime}\right.$, where $\left.\tau \ll \tau_{\text {dwell }} \ll \tau^{\prime}\right)$. (Physically, this window is of interest because it contains those resonances that determine the mesoscopic fluctuations of cross sections when the system is probed via the openings, as well as directional lasing modes in dielectric microresonators [9].) We arrive at this conclusion by a combination of semiclassical arguments (based on a tailor-made phase-space representation of reso-
PACS number(s): 05.45.Mt, 03.65.Sq

nance wave functions, which avoids problems with their mutual nonorthogonality) with numerical results for a paradigmatic quantum-dynamical model system, the open kicked rotator, [4,10-12], which can be realized for atoms in pulsed optical fields [13].

\section{HUSIMI-SCHUR REPRESENTATION}

We start our considerations with some general observations about quantum-to-classical correspondence. A basic ingredient in the derivation of the ordinary Weyl law in closed systems is the mutual orthogonality of energy eigenstates, which is guaranteed by the Hermiticity of the Hamiltonian (equivalently, the unitarity of the time-evolution operator). Quantum-to-classical correspondence can then be exploited, e.g., by using a basis of semiclassically localized states $|x\rangle$ which occupy Planck cells in phase space $x=(\mathbf{q}, \mathbf{p})$. Resonance wave functions, however, overlap with each other because open systems are necessarily represented by nonnormal (neither Hermitian nor unitary) operators. In open quantum maps, for example, resonances are associated to the spectrum of a truncated unitary matrix $\mathcal{M}=\mathcal{Q F Q}$, composed of the unitary time-evolution operator $F$ of the closed system and the projector $\mathcal{Q}=\mathcal{Q}^{2}$ onto the nonleaky part of the Hilbert space (see Fig. 1 for an example of an open quantum map). Because of the truncation, the eigenvalues $\mu_{n}=\exp \left(-i E_{n}-\gamma_{n} / 2\right)$ of $\mathcal{M}$ lie inside the unit disk of the complex plane, ensuring that the decay rates $\gamma_{n}=1 / \tau_{n}$ are positive [14]. But since $\mathcal{M}$ is not normal, the associated eigenstates-which now describe the resonance wave functions-are not orthogonal to each other. This circumstance, which is the root for the scarceness of analytical tools in non-normal problems, complicates the task of exploiting quantum-to-classical correspondence for the purpose of resonance counting.

We circumvent this problem by applying standard phasespace methods to an alternative spectral decomposition, the Schur decomposition $\mathcal{M}=U T U^{\dagger}$, which delivers the same eigenvalues (occupying the diagonal of the upper triangular matrix $T$ ) but associates to them an orthogonal basis set $U$ [15]. The Schur decomposition exists for any operator, even when it is not normal; moreover, this decomposition remains stable against small perturbations. For definiteness assume that all eigenvalues are ordered by their modulus, $\left|\mu_{1}\right| \leq\left|\mu_{2}\right| \leq\left|\mu_{3}\right| \leq \cdots \leq\left|\mu_{M}\right|$. The first $r$ rows $u_{r}$ of $U$ then form a complete basis for the $r$ fastest decaying resonance 
+ states. Quantum-to-classical correspondence can now be exploited by considering the Husimi representation [16] $\mathcal{H}_{r}(x)=\sum_{m=1}^{r}\left|\left\langle x \mid u_{m}\right\rangle\right|^{2}=\left\langle x\left|\Pi_{r}\right| x\right\rangle$ of this subspace, where $\Pi_{r}=\sum_{m=1}^{r}\left|u_{m}\right\rangle\left\langle u_{m}\right|$ is the associated projector. This provides insight into the regions in classical phase space which support the quickly decaying quantum resonances. An analogous construction can be based on the opposite ordering, $\left|\mu_{1}\right| \geq\left|\mu_{2}\right| \geq\left|\mu_{3}\right| \geq \cdots \geq\left|\mu_{M}\right|$, which focuses on the slowest decaying resonance wave functions. Because of nonorthogonality, the resulting "fast" and "slow" Husimi-Schur representations carry independent information on the resonance wave functions.

Figure 1 demonstrates the viability of this method for a paradigm of quantum chaos, the kicked rotator [10] with unitary time-evolution operator,

$$
\begin{aligned}
F_{n m}= & (i M)^{-1 / 2} \\
& \times \exp \left[\frac{i \pi}{M}(m-n)^{2}-\frac{i M k}{4 \pi}\left(\cos \frac{2 \pi n}{M}+\cos \frac{2 \pi m}{M}\right)\right],
\end{aligned}
$$

which classically reduces to the (symmetrized) standard map $p^{\prime}=p+k \cos (2 \pi q+\pi p) \bmod 1, \quad q^{\prime}=q+p / 2+p^{\prime} / 2 \bmod 1$. The matrix dimension $M=h^{-1}$ determines the inverse effective Planck's constant [17] (corresponding to discrete initial and final positions $\left.q=m / M, q^{\prime}=n / M\right)$, while the kicking strength $k$ determines the nonlinearity. For $k=0$ the classical system is integrable, while for $k \gtrsim 7$ it is globally chaotic (resonances in the chaotic variant have been studied in Refs. $[4,12])$.

In Fig. 1, the kicking strength is set to $k=2$, for which the phase space of the closed system is mixed, as shown by trajectory segments (black dots) in Fig. 1(a). The color-coded areas superimposed on the phase space indicate the classical initial conditions for escape after one, two, three, or four iterations when an opening is placed at $0<q<0.2$ $\left(\tau_{\text {dwell }}=5\right)$. Figure $1(\mathrm{~b})$ shows the ordinary Husimi representation of quickly decaying resonance eigenfunctions with $0<\left|\mu_{n}\right|<0.1(M=1280)$. Figures 1(c) and 1(d) show the fast Husimi-Schur representation $\left(0<\left|\mu_{n}\right|<0.1 ; M=160\right.$ and $M=1280$ ), while Figs. 1(e) and 1(f) display the slow HusimiSchur representation $\left(0.98<\left|\mu_{n}\right|<1 ; M=160\right.$ and $\left.M=1280\right)$ [18].

The ordinary Husimi representation demonstrates that the quickly decaying resonance eigenfunctions are all localized in the region of escape after a single iteration. However, since these eigenfunctions strongly overlap, partitioning their phase-space support with Planck cells significantly underestimates their number (predicting $\simeq 25$ instead of $r=44$ short-lived states for $M=160$, and $\simeq 200$ instead of $r=568$ short-lived states for $M=1280$ ). In contrast, the fast HusimiSchur representation clearly maps out the classical escape zones and uncovers that the domain of quantum-to-classical correspondence increases with increasing $M$. The slow Husimi-Schur representation maps out stable phase-space regions that are classically decoupled from the opening by impenetrable dynamical barriers; these regions do not significantly change as $M$ increases.

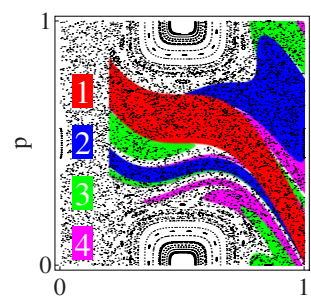

(a)

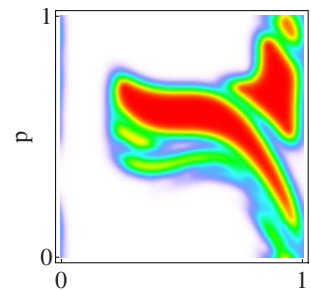

(c)

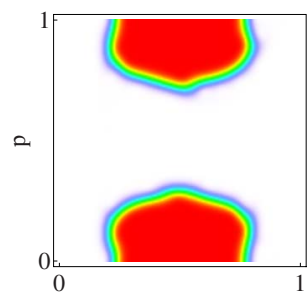

(e)

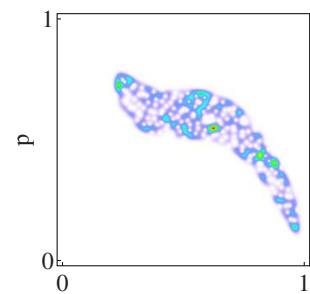

(b) q

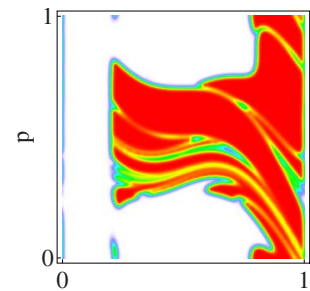

(d) $\quad$ q

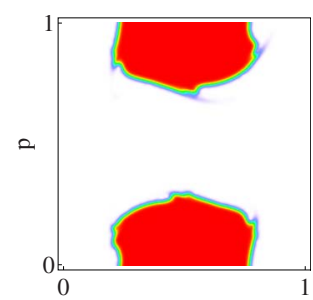

(f)
FIG. 1. (Color online) Quantum versus classical escape in a kicked rotator (kicking strength $k=2.0$ ) with opening at $0<q<0.2$. (a) Classical escape zones in phase space $(q, p)$, color coded according to escape after one (red), two (blue), three (green), or four (magenta) iterations (for gray scale see the markers in the figure). The black dots are trajectory segments in the closed system, whose phase space is a mixture of regular and chaotic motion. (b) Husimi representation of short-lived quantum resonances with decay time $\tau_{n}<1 /(2 \ln 10)$ (decay factor $\left|\mu_{n}\right|<0.1$ ), for Hilbert-space dimension $M=1280$. The other panels show the fast [panels (c) and (d), $\left|\mu_{n}\right|<0.1$ ] and slow [panels (e) and (f), $\left|\mu_{n}\right|>0.98$ ] HusimiSchur representation, introduced in this work, for $[(\mathrm{c})$ and $(\mathrm{e})]$ $M=160$ and [(d) and (f)] $M=1280$.

By construction, Planck-cell partitioning of the support of the Husimi-Schur representations accurately estimates the underlying number of resonances. The fast Husimi representation therefore uncovers a proliferation of anomalously short-lived states driven by the emerging quantum-toclassical correspondence-among the total count of $M$ resonances, the fraction of short-lived states increases as $M$ increases. On the other hand, the slow Husimi representation shows that the fraction of anomalously long-living resonances supported by classically uncoupled regions remains fixed, which corresponds to the ordinary Weyl law for this effectively closed-off part of the system. Crucially, we are led to conclude that the remaining fraction of resonances with intermediate lifetime (chosen here to satisfy $0.1<\left|\mu_{n}\right|<0.98$ ) decreases as $M$ increases, and therefore cannot follow an ordinary Weyl law. (The absolute number of such resonances still increases with increasing $M$.) 

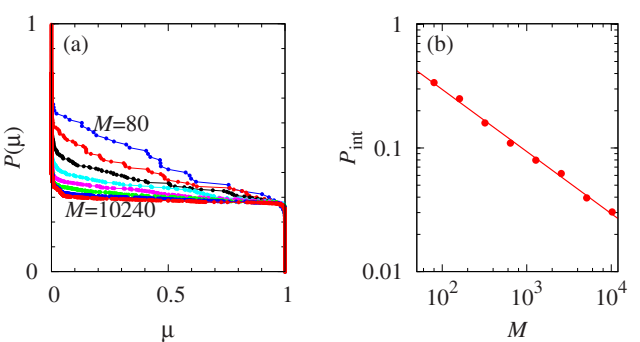

FIG. 2. (Color online) Fractal Weyl law for resonances in the open kicked rotator with a mixed classical phase space (kicking strength $k=2.0$, opening at $0<q<0.2$ ). (a) Fraction $P(\mu)$ of resonances with decay factor $\left|\mu_{n}\right|>\mu$, corresponding to a lifetime $\tau_{n}>-1 /(2 \ln \mu)$. (b) Fraction $P_{\text {int }}$ of resonances in a band of intermediate lifetimes $\left(0.1<\left|\mu_{n}\right|<0.98\right)$, as a function of dimensionless system size $M$. In this double-logarithmic representation, the fractal Weyl law results in a linear dependence with a negative slope.

\section{FRACTAL WEYL LAW}

A detailed understanding of the resonance distribution can be obtained by counting the resonances in fixed lifetime windows and comparing these counts for different values of $M$. We adopt probabilistic terminology and proceed in two steps. In the first step we determine the fraction $P(\mu)=\operatorname{prob}\left(\left|\mu_{n}\right|>\mu\right)$ of resonances with lifetime exceeding a lower threshold $\tau=-1 /(2 \ln \mu)$. This defines a monotonously decreasing function interpolating between $P(0)=1$ and $P(1)=0$. In the second step, we extract the fraction of resonances within an interval of intermediate lifetimes $\quad\left(0.1<\left|\mu_{n}\right|<0.98\right)$, which follows from $P_{\text {int }}=\operatorname{prob}\left(\left|\mu_{n}\right| \in[0.1,0.98]\right)=P(0.1)-P(0.98) \quad$ (our results and conclusions do not depend on the chosen window as long as it stays in the range of typical classical lifetimes).

Numerical results for the system with opening at $0<q<0.2$ are shown in Fig. 2. Figure 2(a) shows $P(\mu)$ for various values of $M$. As a function of $\mu, P$ decreases very sharply at the two extreme ends of the graph. At $\mu \simeq 0$ we witness the influence of extremely short-lived resonances, while at $\mu \simeq 1$ we observe the states which have very long lifetimes. The applicability of the ordinary Weyl law would entail that modulo small fluctuations, $P(\mu)$ is independent of $M$, since the uncertainty-limited resolution of phase space increases uniformly when the Planck cell shrinks. The plot, however, shows that the body of the function $P$ drops as $M$ increases. This is due to the proliferation of the short-lived resonances $(\mu \simeq 0)$, whose relative fraction among all resonances increases with increasing $M$, in agreement with the expanding domain of support of the fast Husimi-Schur representation. In the region of long-living states $(\mu \simeq 1)$, on the other hand, $P$ does not depend significantly on $M$, in agreement with the observed $M$-independent support of longliving resonances in the slow Husimi-Schur representation.

Complementing these trends for short and long lifetimes, the body of $P$ becomes flatter as $M$ increases. As shown in Fig. 2(b), the fraction $P_{\text {int }}$ of resonance with intermediate lifetime therefore decreases with increasing $M$. The linear fit in this double-logarithmic plot demonstrates that this trend closely follows a power law, $P_{\text {int }} \propto M^{-0.50}$. These resonances therefore obey a fractal Weyl law, $\mathcal{N}_{\text {int }}=M P_{\text {int }} \propto M^{0.50}$.
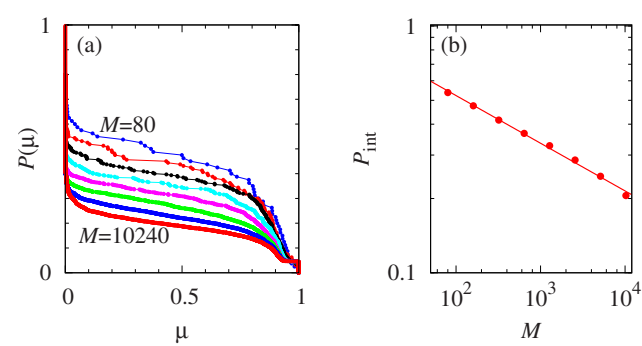

FIG. 3. (Color online) Same as Fig. 2, but with opening shifted to $0.2<q<0.4$.

Figure 3 shows that the fractal Weyl law remains intact when the opening is shifted to $0.2<q<0.4$, so that it couples to a larger part of the regular regions in phase space [see Fig. 4(a)]. In this case $P_{\text {typ }} \propto M^{-0.19}$, and therefore $\mathcal{N}_{\text {typ }}$ $\propto M^{0.81}$. Compared to the situation in Fig. 2, the fraction of long-living states is now reduced, in keeping with the shrunken size of the classically uncoupled phase-space region. The fast Husimi-Schur representation [Fig. 4(b)] shows that the newly coupled parts support additional short-lived resonances. Consequently, the states with intermediate lifetime are still associated to the chaotic regions. We also studied other values of $k$ and positions of the opening (see the Appendix) and always found that a fractal Weyl law holds true.

\section{DISCUSSION AND CONCLUSIONS}

For globally chaotic systems, the fractal Weyl law can be understood by associating the proliferation of short-lived resonance to quasideterministic decay following classical escape routes [4]. This quasideterministic decay requires classical-to-quantum correspondence, which is lost exponentially on a time scale given by the Ehrenfest time $\tau_{E}$ $=\lambda^{-1} \ln M$ [19]. The probability to reside within the system decays exponentially, too, and is governed by $\tau_{\text {dwell }}$. The power law for the fractal Weyl law therefore arises from the combination of two exponential laws, based on the relation $\exp \left(-\tau_{E} / \tau_{\text {dwell }}\right)=M^{-1 / \lambda} \tau_{\text {dwell }}$ for the part of phase space where classical-to-quantum correspondence does not apply; this re-

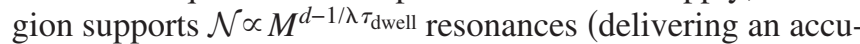
rate estimate for $d_{H}$ if the opening is sufficiently small [7]).

For the kicked rotator with a mixed phase space, our Husimi-Schur representation confirms the association of

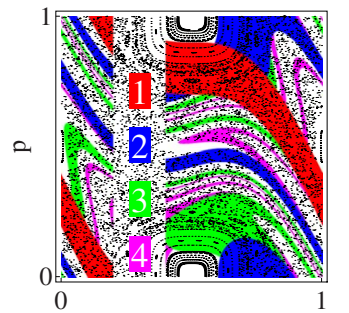

(a) q

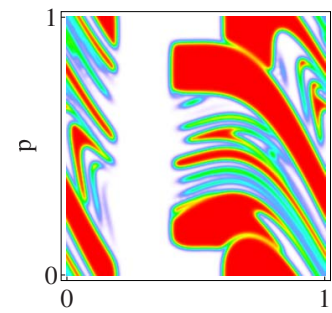

(b)

q
FIG. 4. (Color online) (a) Classical escape zones and (b) fast Husimi-Schur representation of short-lived quantum resonances $(M=1280)$ for the kicked rotator in Fig. 1, with opening shifted to $0.2<q<0.4$. 
short-lived resonances to quasideterministic escape routes, while the resonances of intermediate lifetime are now associated to chaotic regions. It is generally accepted that these regions are characterized by classical power-law decay $\propto t^{-\alpha}$ in place of the exponential decay [20], while there is also evidence that loss of quantum-to-classical correspondence in these regions is similarly modified into a power law $\propto t^{\beta}$, so that the Ehrenfest time takes the algebraic form $\tau_{E} \propto M^{1 / \beta}$ [21]. Heuristically, therefore, it is tempting to suggest that the fractal Weyl law now arises from the combination of two power laws, based on the relation $\tau_{E}^{-\alpha} \propto M^{-\alpha / \beta}$ for the part of phase space where quantum-to-classical correspondence does not apply. For individual realizations as studied here, however, the classical escape is characterized by multiscale decay, displaying varying exponents $\alpha$ associated to switching between island structures explored at different times [22]. Over the computationally accessible range, we find that the fractal Weyl law is always characterized by a single exponent (see also the results in the Appendix), which would indicate that any multiscale fluctuations of the decay (exponent $\alpha$ ) are compensated by multiscale fluctuations in the loss of quantum-to-classical correspondence (exponent $\beta$ ).

In summary, we have demonstrated that open quantum systems with mixed phase space can obey a modified fractal Weyl law, even when the classical dynamics displays a mixed phase space. We unraveled this law by introducing the concept of a Husimi-Schur representation, a phase-space representation of resonance wave functions which captures maximal information on quantum-to-classical correspondence (circumventing the problem that resonance eigenfunctions are not orthogonal to each other). The formation of anomalously short-lived states that drive the departure from the ordinary Weyl law originates in quasideterministic decay along classical escape routes, whose phase-space support expands as one approaches the classical limit.
The kicked rotator used here for the illustration can be realized with atoms that are driven by pulsed optical waves [13]. Experimentally, direct evidence of the fractal Weyl law is more likely to come from autonomous (nondriven) systems, which can be related to quantum maps via surface of section methods [23]. In particular, advanced techniques now enable the accurate determination of complex resonance frequencies in microwave resonators [24]; a mixed phase space is obtained for any generic smooth resonator shape.

\section{ACKNOWLEDGMENT}

This work was supported by the European Commission via Marie Curie Excellence Grant No. MEXT-CT-2005023778 .

\section{APPENDIX: NUMERICAL RESULTS FOR OTHER KICKING STRENGTHS}

In our computations for the open kicked rotator we find that the fractal Weyl law for resonances with intermediate lifetime holds robustly when the kicking strength or the opening position is varied. Figures 5 illustrates these findings for kicking strength $k=2.5$, where the main stability island has four large satellites (see phase-space portrait in Fig. 6). For comparison, we also present in Fig. 7 results for an almost-chaotic phase space with small islands $(k=5.5)$, and in Fig. 8 results for the case $k=7.5$ of a strongly chaotic system. In all cases the fast Husimi-Schur representation demonstrates that the fractal Weyl law is associated to the expanding domain of quantum-to-classical correspondence. This substantiates the link to the fractal repeller at the scale of the Ehrenfest time.
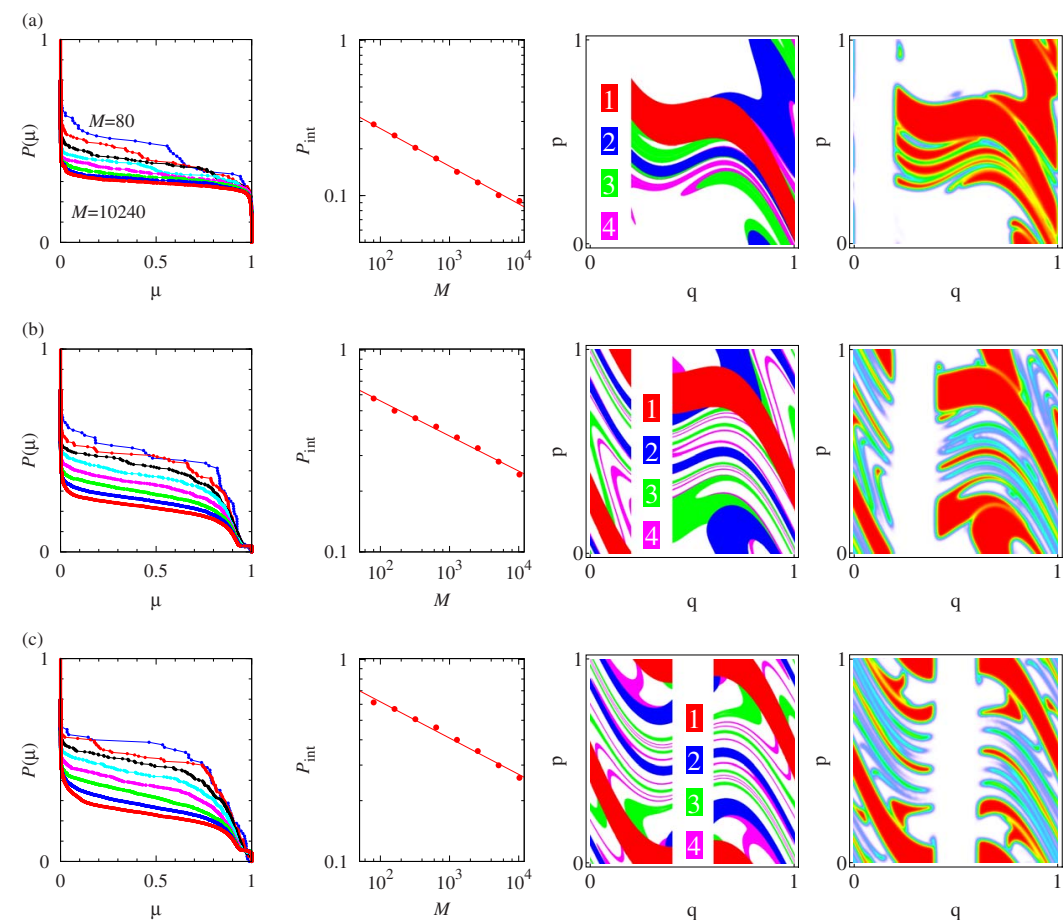

FIG. 5. (Color online) Fractal Weyl law for resonances in the open kicked rotator with $k$ $=2.5$ and various positions of the opening: (a) opening at $0<q<0.2$; (b) opening at $0.2<q$ $<0.4$; (c) opening at $0.4<q<0.6$. First column: fraction $P(\mu)$ of resonances with decay factor $\left|\mu_{n}\right|>\mu$, corresponding to a lifetime $\tau_{n}>$ $-1 /(2 \ln \mu)$. Second column: fraction $P_{\text {int }}$ of resonances in a band of intermediate lifetimes $\left(0.1<\left|\mu_{n}\right|<0.98\right)$, as a function of dimensionless system size $M$. In this double-logarithmic representation, the fractal Weyl law results in a linear dependence with a negative slope. Third column: classical escape zones. Last column: fast HusimiSchur representation of short-lived quantum resonances $(M=1280)$. 

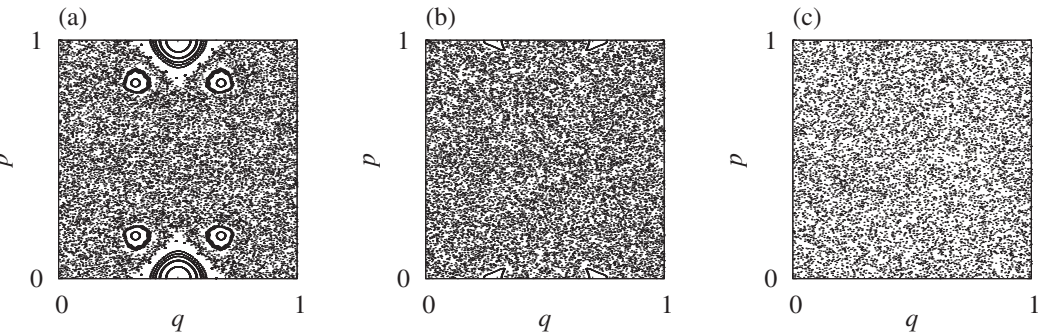

FIG. 6. Phase-space portraits of the kicked rotator with kicking strengths (a) $k=2.5$, (b) $k=5.5$, and (c) $k=7.5$.
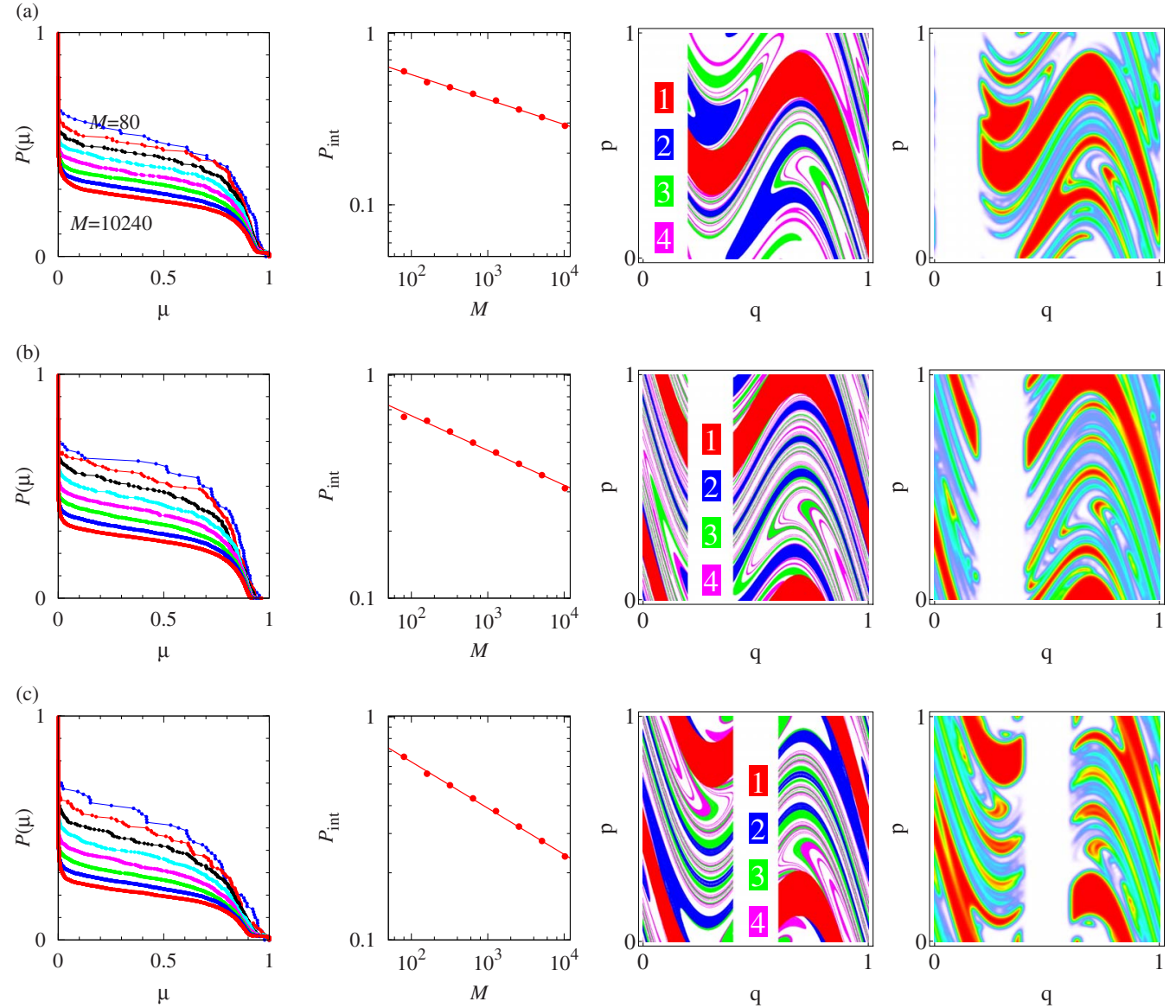

FIG. 7. (Color online) Same as Fig. 5, but for kicking strength $k=5.5$.
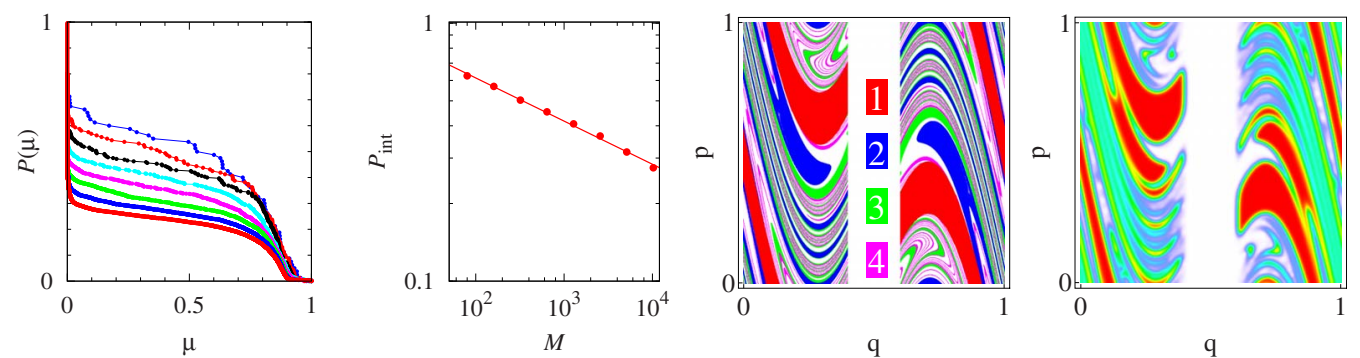

FIG. 8. (Color online) Same as Fig. 5, but for kicking strength $k=7.5$, for which the system is strongly chaotic (since the phase space is globally chaotic, results are shown for only one position of the opening $(0.4<q<0.6)$; see also Ref. [4]). 
[1] P. M. Morse and K. U. Ingard, Theoretical Acoustics (Princeton University Press, Princeton, NJ, 1968).

[2] H. P. Baltes and E. R. Hilf, Spectra of Finite Systems (B-I Wissenschaftsverlag, Mannheim, 1978).

[3] W. T. Lu, S. Sridhar, and M. Zworski, Phys. Rev. Lett. 91, 154101 (2003).

[4] H. Schomerus and J. Tworzydło, Phys. Rev. Lett. 93, 154102 (2004).

[5] S. Nonnenmacher and M. Zworski, J. Phys. A 38, 10683 (2005).

[6] J. P. Keating, M. Novaes, S. D. Prado, and M. Sieber, Phys. Rev. Lett. 97, 150406 (2006).

[7] E. Ott, Chaos in Dynamical Systems (Cambridge University Press, Cambridge, England, 1993).

[8] O. Bohigas, S. Tomsovic, and D. Ullmo, Phys. Rep. 223, 43 (1993).

[9] C. Gmachl et al., Science 280, 1556 (1998).

[10] F. M. Izrailev, Phys. Rep. 196, 299 (1990).

[11] H. Schomerus and Ph. Jacquod, J. Phys. A 38, 10663 (2005).

[12] D. L. Shepelyansky, Phys. Rev. E 77, 015202(R) (2008).

[13] F. L. Moore, J. C. Robinson, C. F. Bharucha, B. Sundaram, and M. G. Raizen, Phys. Rev. Lett. 75, 4598 (1995).

[14] The projector on the leaky part is $\mathcal{P}=1-\mathcal{Q}$, and rank $\mathcal{P}=N$ determines the number of open decay channels. Since $\mathcal{M P}$ $=0$, there are also $N$ trivial eigenvalues with $\mu_{n}=0$ and therefore $\tau_{n}=0$, corresponding to states that immediately decay through the opening.
[15] G. H. Golub and C. F. van Loan, Matrix Computations, 3rd ed. (Johns Hopkins University Press, Baltimore, MD, 1996).

[16] H. W. Lee, Phys. Rep. 259, 147 (1995).

[17] This constitutes the Weyl law for the closed system.

[18] In Figs. 1(b)-1(d) and 4(b) we omit for clarity the $N$ trivial eigenvalues with $\mu_{n}=0$, which uniformly cover the opening.

[19] This can be quantified using the propagation of semiclassical projectors $\widetilde{\Pi}_{t}$ onto the zones of escape after $t$ iterations. Under backward-in-time propagation with the truncated unitary timeevolution operator $\mathcal{M}^{\dagger}, \mathcal{M}^{\dagger s} \widetilde{\Pi}_{t} \mathcal{M}^{s}=\widetilde{\Pi}_{t+s}+R(t)$, where the remainder $R(t)$ has exponential or algebraic time dependence depending on the nature of the classical motion, and becomes non-negligible at $t \approx \tau_{E}$.

[20] G. Cristadoro and R. Ketzmerick, Phys. Rev. Lett. 100, 184101 (2008).

[21] S. Fishman, D. R. Grempel, and R. E. Prange, Phys. Rev. A 36, 289 (1987).

[22] M. Weiss, L. Hufnagel, and R. Ketzmerick, Phys. Rev. E 67, 046209 (2003).

[23] E. B. Bogomolny, Nonlinearity 5, 805 (1992); B. Georgeot and R. E. Prange, Phys. Rev. Lett. 74, 4110 (1995); R. O. Vallejos and A. M. Ozorio de Almeida, Ann. Phys. (N.Y.) 278, 86 (1999).

[24] U. Kuhl, R. Höhmann, J. Main, and H.-J. Stöckmann, Phys. Rev. Lett. 100, 254101 (2008). 